\title{
THE OPERATION OF A PRESSURIZED ULTRAVIOLET PHOTOIONIZATION THRESHOLD CHERENKOV COUNTER *
}

\author{
N. HARNEW and D.I. MEYER \\ The University of Michigan, Department of Physics, Ann Arbor, MI 48109, U.S.A.
}

Received 19 January 1981

We have successfully tested an ultraviolet photoionization Cherenkov counter in a $10 \mathrm{GeV} / c$ pion beam. The counter has been tested to $11 \mathrm{~atm}$ pressure for use as a $\pi-\mathrm{K}$ separator. The design and operation of the counter is described.

\section{Introduction}

The importance of kaon identification over a large solid angle at $\mathrm{e}^{+} \mathrm{e}^{-}$storage rings has long been realized at PEP and PETRA energies. The identification of a kaon can be the signature for charm decay which in turn can lead to reconstruction of the B meson. It can also prove invaluable in any detailed jet physics study. However, despite high machine energies, high multiplicities imply that the large majority of particles have low to medium energies. A large fraction are in the 1-2 $\mathrm{GeV} / c$ momentum range. Unfortunately this is the very range that is difficult for timeof-flight and $\mathrm{d} E / \mathrm{d} x$ techniques. The tight bunching of particles in jets compounds the difficulty of identification.

A useful device to fill this gap would be a pressurized threshold Cherenkov counter. However, the need to keep the phototubes of a conventional counter outside any magnetic detector makes a light guide system difficult if not impossible to design, especially for a highly segmented system. For this reason, Cherenkov counters which employ the far ultra- violet part of the Cherenkov spectrum (1150-1350 $\AA$ ) have been studied $[1,2]$. To detect the light a proportional chamber doped with a small amount of benzene is used. The benzene has a high photoionization probability in this wavelength range. Tests showed that highly efficient counters (operating at atmospheric pressure with argon or nitrogen radiators) can be built [1] which provide excellent $\pi-$ separation up to $4 \mathrm{GeV} / c$ momentum. A pressurized counter

* Supported by the U.S. Department of Energy. using this same principle of operation should allow pion identification in the important $1-2 \mathrm{GeV} / c$ momentum region.

The present paper describes tests of a prototype cell of an ultra-violet threshold Cherenkov counter system which we plan to construct for the High Reso. lution Spectrometer (HRS) at PEP. The counters will consist of 13 tori placed around the beam as shown in fig. 1. The design allows for high segmentation ( 64 azimuthal sections) and will at the same time minimize the amount of material contributing to multiple Coulomb scattering. The Cherenkov radiator will be an argon--nitrogen mixture at 16 atm pressure which gives a $\pi$ threshold of $1.1 \mathrm{GeV} / c$ and will allow $\pi / K$ separation from 1.1 to $3.9 \mathrm{GeV} / c$. Time of flight counters will be used for particle identification at lower momenta.

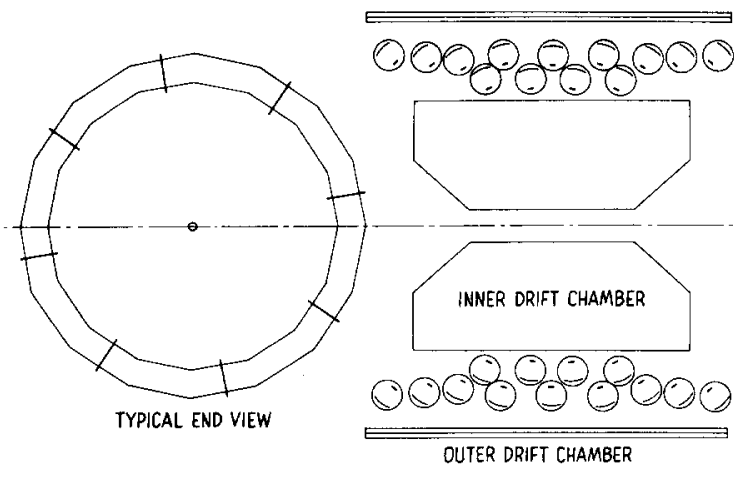

Fig. 1. End and side view of planned Cherenkov counter system for the HRS. 


\section{Choice of radiator}

The number of photons per unit length of Cherenkov radiator is given by the expression

$\mathrm{d} \phi / \mathrm{d} l=370 \int \epsilon \sin ^{2} \theta \mathrm{d} E$,

where $l$ is the particle path length in $\mathrm{cm}, \theta$ is the Cherenkov angle, $E$ is the photon energy in $\mathrm{eV}$ and $\epsilon$ is the photon detection efficiency. $\epsilon$ is dependent on the benzene photoionization efficiency, $\mathrm{MgF}_{2}$ window transmission and mirror reflectance, all of which vary with photon energy [1]. Also affecting $\epsilon$ is the percentage of $\mathrm{CO}_{2}$ which is used as a quenching gas in proportional chambers since it is absorbing below $1160 \AA$. A further possible factor is the absorption of photons in the Cherenkov radiator which could reduce the photon yield, especially in a pressurized system.

There is no gas which has a high enough refractive index to allow $\pi / \mathrm{K}$ separation down to $1 \mathrm{GeV} / \mathrm{c}$ at atmospheric pressure. In fact the only gases known to transmit within the desired wavelength range are nitrogen and the noble gases. It is well known that argon is transparent to photons in the 1150-1350 $\AA$ wavelength range and this makes it a natural choice for use as a Cherenkov radiator. However the refractive index of argon is such that a 16 atm Cherenkov counter must be constructed for the desired particle

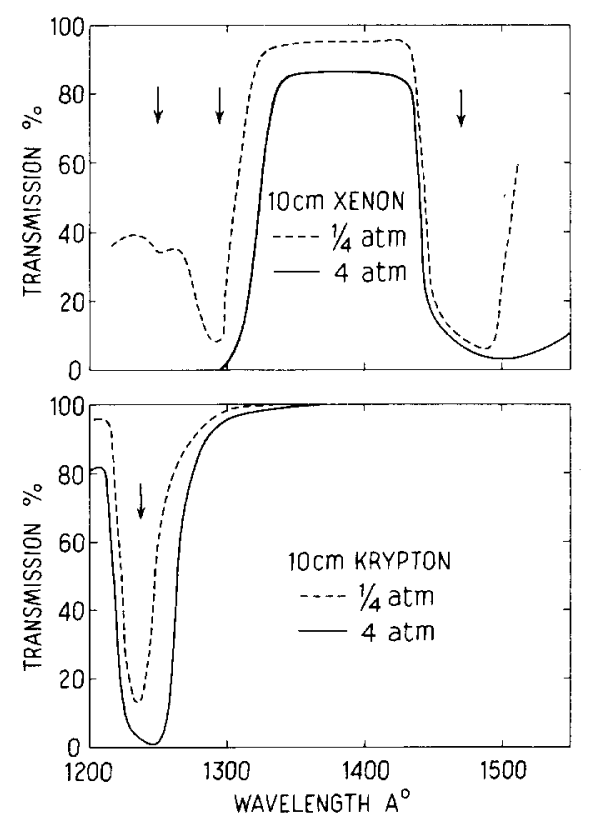

Fig. 2. Absorption spectra of xenon and krypton. Arrows represent position of spectral lines. separation. Also argon is known to scintillate at these wavelengths so a suitable quenching gas must be found. Another possible candidate is nitrogen. Although this has a similar refractive index to argon, it is known to absorb ultra-violet photons weakly [3] . Although the absorption length is large $(187 \mathrm{~cm})$, the effect is significant for a high pressure counter. Other possible candidates are krypton and xenon which by extrapolation from the visible probably have higher indices of refraction than argon and nitrogen [4] although they have not been measured at these wavelengths. They both have excited states and hence absorption lines in the region of interest.

We have measured the transmission of xenon and krypton with an ultraviolet spectrometer at moderate pressures. The results are shown in fig. 2 . It can be seen that xenon is transparent between 1320 and $1440 \AA$ and krypton is transparent above $1250 \AA$. Note however, the broadening of the absorption lines even at $4 \mathrm{~atm}$. This will undoubtedly become worse at higher pressures. Triethylamine has a high photoionization efficiency around $1500 \AA$ [5] and therefore might be a suitable chemical to use with xenon. However we decided not to pursue the use of xenon as we believe the photon yield would be inadequate. Krypton would give an adequate photon yield however the disadvantage of its high cost outweighed the advantage over argon of its slightly higher refractive index. In addition the krypton scintillation light was difficult to handle. We therefore decided that argon, doped with a small amount of nitrogen to quench scintillation light was the most suitable radiator gas.

\section{Experimental setup}

A prototype of the cell geometry to be used in the HRS was built. This geometry is shown in fig. 3 . The test section consisted of a $25.4 \mathrm{~cm}$ diameter aluminum tube of $0.32 \mathrm{~cm}$ thickness. Contained inside the tube was a $22.9 \mathrm{~cm}$ wide by $17.8 \mathrm{~cm}$ long elliptical mirror with a cylindrical cross section. The proportional chamber was placed $15 \mathrm{~cm}$ from the mirror at its first focus. The second focus would correspond to the $\mathrm{e}^{+} \mathrm{e}^{-}$intersection point, $200 \mathrm{~cm}$ away.

The structure of the proportional chamber is shown in fig. 4 . The chamber was $15.2 \mathrm{~cm}$ long and contained six cells, each $0.5 \mathrm{~cm}$ wide. The dead region between cells was $0.5 \mathrm{~mm}$. Two $1.75 \mathrm{~cm}$ wide $\mathrm{MgF}_{2}$ windows, $5 \mathrm{~mm}$ thick were epoxied together lengthways. The epoxy joint between them caused a 


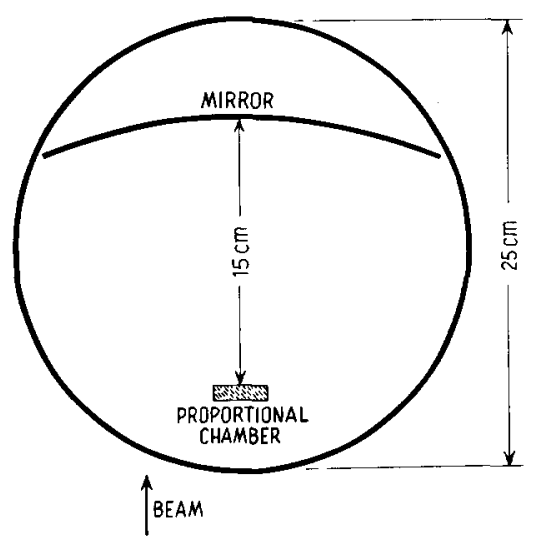

Fig. 3. Cross sectional view of Cherenkov counter test vessel.

loss of photons, however these windows were the only ones available to us at that time. A single $2 \mathrm{~mm}$ thick window has been tested and is being used in the final design. The six cell structure was chosen for two reasons. The proportional chamber runs at atmospheric pressure and the outside medium is at $16 \mathrm{~atm}$. Hence the chamber structure must support the $\mathrm{MgF}_{2}$ window. Also the chamber presents a dead region to particles since if a particle goes through the proportional chamber it will fire regardless of whether the particle was above threshold or not. If the chamber has discrete cells, the cell the particle went through can be ignored and the particle idenitified by looking for Cherenkov light in the other 5 cells.

The design of the proportional chamber has gone through a variety of stages. We have tested a two stage chamber proposed by Charpak et al. [6] but concluded the design was too complicated and costly

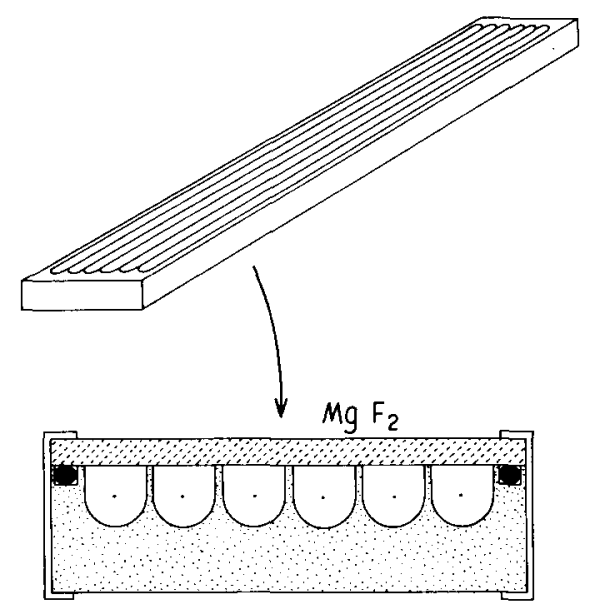

Fig. 4. Structure of proportional chamber. for our proposed system. We eliminated the wire grid just under the window used previously to shape the electric field in the proportional chamber [1]. The grid made the construction of the chambers more complicated, intercepted some photons and had negligible effect on the single photon detection efficiency.

We found that adding benzene as a photoionizing chemical tends to destabilize the proportional chamber. When a proportional avalanche occurs many argon atoms are excited. The argon emission spectrum is centered around $1000 \AA$. These photons can photoionize the benzene causing further avalanches. A suitable quenching agent must absorb these photons while transmitting UV light in the 1350-1150 \& region. $\mathrm{CO}_{2}$ has a short absorption length below $1160 \AA$ and a "window" at the desired wavelengths [7]. We experimented with a number of gas mixtures - argon with $1 \% \mathrm{C}_{6} \mathrm{H}_{6}$ and $5 \%, 10 \%, 20 \%$, and $30 \% \mathrm{CO}_{2}$. We have found that more $\mathrm{CO}_{2}$ is needed than for a standard proportional chamber, a useful mixture being $1 \%$ benzene, $20 \% \mathrm{CO}_{2}$ and $79 \%$ argon. It was found that $30 \% \mathrm{CO}_{2}$ raised the operating voltage by $100 \mathrm{~V}$ but did not affect the chamber stability or improve the single photon detection efficiency. The $1 \mathrm{~mol} \%$ benzene was chosen so that there are several mean absorption lengths for the UV photons in the chamber. The sense wire (38 $\mu \mathrm{m}$ diameter goldplated tungsten) was positioned $2.4 \mathrm{~mm}$ from the window.

An interesting feature of the proportional chamber was its ability to detect visible photons as evidenced by its sensitivity to an overhead room light. We attributed this to the photoelectric effect of the aluminum oxide layer on the chamer walls. Silvering the proportional chamber surface eliminated the effect.

One worry was that the proportional avalanches would affect window transmission. The transmission of the $\mathrm{MgF}_{2}$ window was measured to be $50 \%$ using a Lyman alpha (1216 $\AA$ ) hydrogen discharge lamp. The chamber was run for $10^{10}$ counts just below breakdown and the $\mathrm{MgF}_{2}$ transmission was remeasured. No noticeable difference was observed indicating that breakdown products formed by counter avalanches do not degrade the window transmission.

The mirror reflectivity has been measured to be $75 \%$ at $1216 \AA$. The mirror was made of glass, $2 \mathrm{~mm}$ thick. It was slumped in a graphite form which had the desired elliptical curvature. The glass was then vacuum aluminized with a $\mathrm{MgF}_{2}$ overcoating to pre. vent oxidation [8]. 

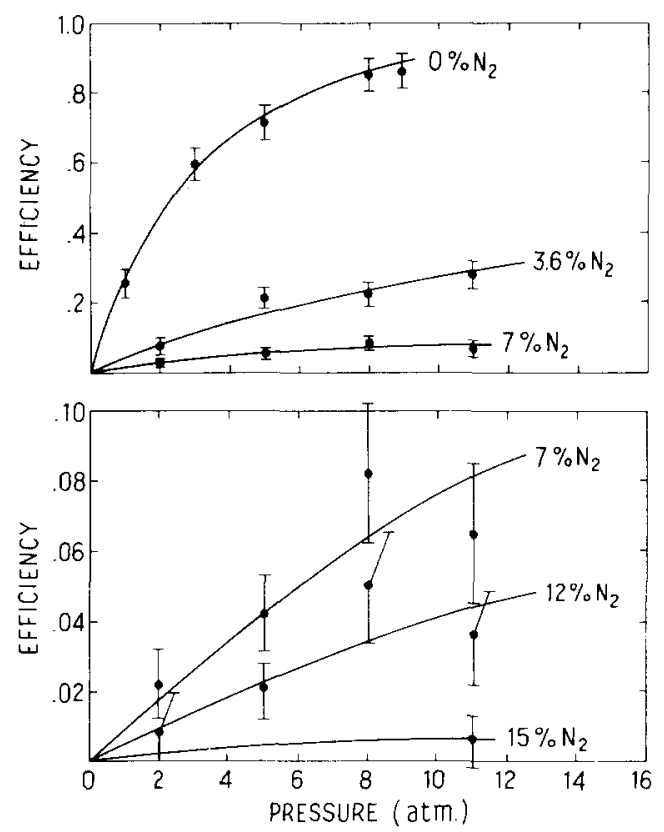

Fig. 5. Efficiency for detecting scintillation light as a function of pressure and nitrogen percentage.

The preamplifier is a crucial element in the system because it must have low noise $\left(3 \times 10^{-16} \mathrm{C}\right)$ to detect single photoelectron avalanches efficiently. However since linearity is not required the preamp can be of very simple design.

Scintillation light can be produced by a particle

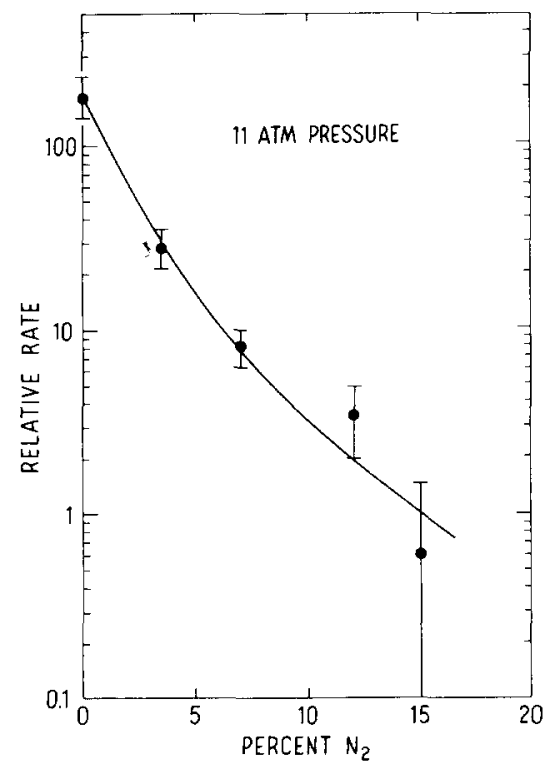

Fig. 6. Scintillation efficiency as a function of nitrogen percentage at 11 atm pressure.

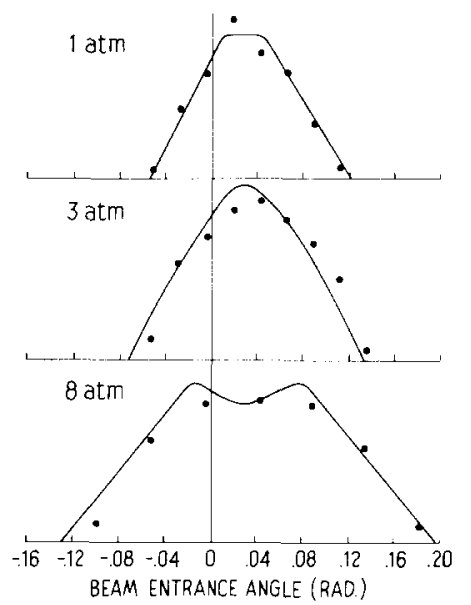

Fig. 7. Measured values and expected values (solid lines) of relative efficiency for detecting Cherenkov radiation as a function of beam direction.

below its Cherenkov threshold destroying the usefulness of the counter for particle identification. Tests were made to determine the scintillation properties of argon-nitrogen mixtures as a function of nitrogen percentage and pressure. We have found that the addition of nitrogen to the argon gas will cause the argon atoms to de-excite before they scintillate. Tests were made to determine what fraction of nitrogen was necessary to keep scintillation light down to an acceptable level. Particles, defined by scintillation counters, went through the Cherenkov radiator at $45^{\circ}$ to the mirror. Under these conditions no Cherenkov light could be reflected from the mirror into the chamber. These tests were made with cosmic rays because of the large $\mu$-meson halo in the test beam.

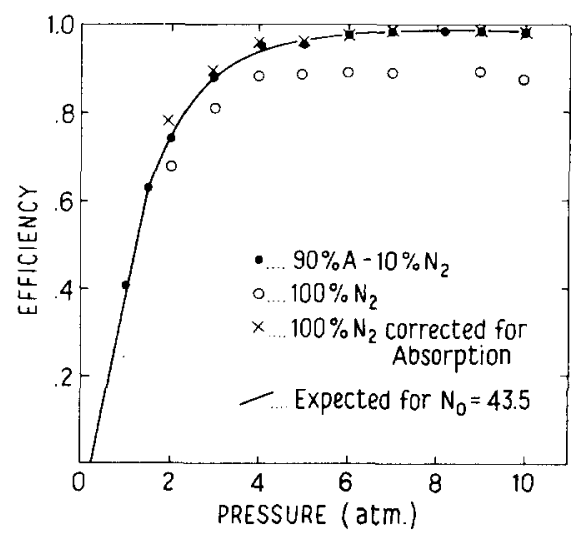

Fig. 8. Efficiency of counter as a function of pressure for various gas mixtures. 


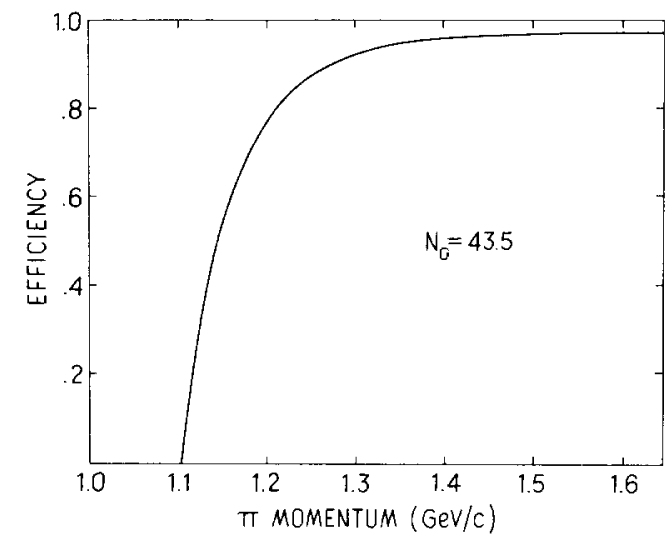

Fig. 9. Expected efficiency of a system of counters as a function of $\pi$ momentum.

Fig. 5 shows the efficiency for detecting particles via scintillation light as a function of pressure for various nitrogen fractions. The values at 11 atm are shown in fig. 6 . It can be seen that an $85 \%$ argon, $15 \%$ nitrogen mixture keeps the scintillation light level below $1 \%$. Although the addition of more nitrogen would further reduce the amount of scintillation light it would also absorb a greater percentage of Cherenkov photons.

We made tests to check the optics of the system using a narrower proportional chamber $1.75 \mathrm{~cm}$ wide. The efficiency of the counter was measured as a function of beam entrance angle with the beam hitting the mirror $5 \mathrm{~cm}$ off axis. The results are shown in fig. 7 at 1,3 , and $8 \mathrm{~atm}$ pressure. The distributions agree with
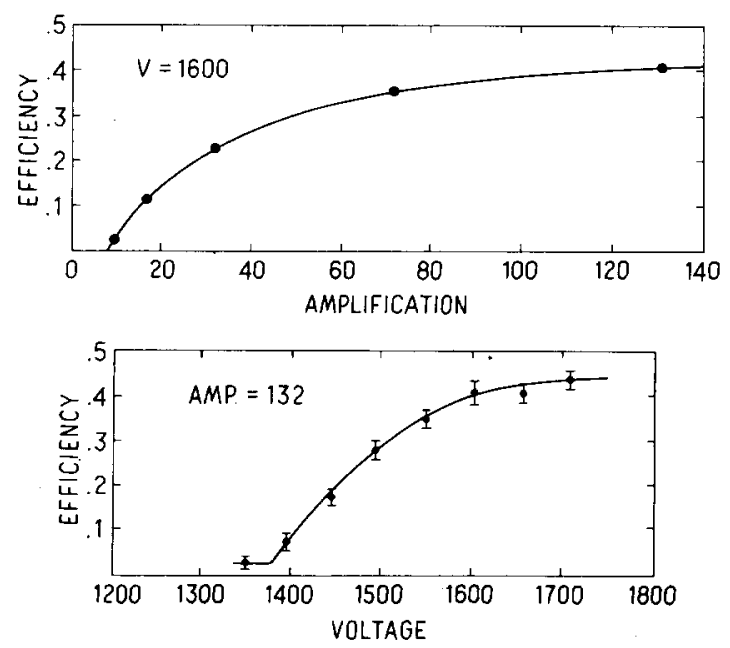

Fig. 10. Relative efficiency as a function of amplification and voltage on the proportional chamber. those expected and confirm that we were observing Cherenkov light. The fact that these distributions drop to zero also confirms that scintillation light is minimal with this gas mixture.

\section{Results}

The Cherenkov counter was tested in a $10 \mathrm{GeV} / c$ negative pion beam at SLAC. The threshold behavior of the counter is shown in fig. 8. Here the efficiency is shown as a function of Cherenkov gas pressure for a $90 \%$ argon-10\% nitrogen and a $100 \%$ nitrogen radiator. The efficiency rises to $98.4 \%$ for the $90 \%$ argon$10 \%$ nitrogen mixture. The small inefficiency is consistent with the antiproton contamination in the beam. Unfortunately there was no low energy beam available at SLAC with which to measure the momentum dependence. The effect of nitrogen absorption is clear. If the nitrogen curve is corrected for absorption in order to agree with the argon curve, the resulting value obtained for the absorption length is within $10 \%$ of the published value [3]. The argon and nitrogen gas used, both had purities of $99.999 \%$ which were sufficient to make absorption by gas impurities unimportant.

Neglecting variation of refractive index with photon energy, the number of photons per unit length of Cherenkov radiator is characterized by the expression $\mathrm{d} \phi / \mathrm{d} l=N_{0} \sin ^{2} \theta$,

where $\theta$ is the Cherenkov angle. The solid curve in fig. 8 which is a fit to the data shows the efficiency expected for $N_{0}=43.5$ based on Poisson statistics. Using this value of $N_{0}$ we can deduce the efficiency of a $16 \mathrm{~atm}$ system proposed for the HRS. Taking into account bending in the magnetic field, finite beam crossing size etc and incorporating the geometry of fig. 1, the efficiency as a function of momentum was evaluated using Monte Carlo techniques. The results of this study are shown in fig. 9. A satisfactory counter can be built with $N_{0}=43.5$. Any improvement in this value will give a steeper threshold behavior.

A calculation based on the efficiency of the system components gives an expected value of $70 \sin ^{2} \theta$ photons per unit length [1]. Although the experimental value of 43.5 is less than expected, fig. 9 demonstrates that a practical system can be built. We believe the difference in measured and expected values to be largely due to the failure to detect small 
pulses associated with single photoelectrons. In a limited test with a lower noise preamplifier and better designed chamber we have attained a voltage plateau for single photoelectrons as shown in fig. 10. This should allow us to attain an $N_{0}$ close to the predicted value in tests which we plan for the near future.

We would like to extend our gratitude to J.W. Chapman for his assistance with the electronics and to both he and $\mathrm{R}$. Thun for more general advice and assistance. Also we would like to thank R. Gearhart and the SLAC Linac operators for their help with the beam.

\section{References}

[1] J. Chapman, D. Meyer and R. Thun, Nucl. Instr. and Meth. 158 (1979) 387.

[2] J. Seguinot and T. Ypsilantis, Nul. Instr. and Meth. 142 (1977) 377.

[3] Y. Tomkiewicz and E. Garwin, Nucl. Instr. and Meth. 114 (1974) 413.

[4] Chaschina et al., Opt. Spect. 24 (1968) 542.

[5] G. Charpak et al., Nucl. Instr. and Meth. 164 (1979) 149.

[6] E.C.Y. Inn, K. Watanabe and M. Zelikoff, J. Chem. Phys. 21 (1953) 1648.

[7] G. Hass and R. Tousey, J. Opt. Soc. 49 (1959) 593. 